\title{
Clinical predictors and explant lung pathology of acute exacerbation of idiopathic pulmonary fibrosis
}

\author{
Yaniv Dotan (1) 1 , William B. Shapiro', Eneida Male1, Eduardo C. Dominguez (1) ${ }^{1}$, \\ Amandeep Aneja ${ }^{2}$, Zhao Huaqing $\mathbb{1}^{3}$, Chandra Dass ${ }^{4}$, Kartik Shenoy ${ }^{1}$, \\ Nathaniel Marchetti ${ }^{1}$, Francis C. Cordova ${ }^{1}$, Gerard J. Criner ${ }^{1}$ and \\ A. James Mamary ${ }^{1}$
}

Affiliations: 'Dept of Thoracic Medicine and Surgery, Lewis Katz School of Medicine at Temple University, Philadelphia, PA, USA. ${ }^{2}$ Pathology and Laboratory Medicine, Lewis Katz School of Medicine at Temple University, Philadelphia, PA, USA. ${ }^{3}$ Dept of Clinical Science, Lewis Katz School of Medicine at Temple University, Philadelphia, PA, USA. ${ }^{4}$ Dept of Clinical Radiology, Lewis Katz School of Medicine at Temple University, Philadelphia, PA, USA.

Correspondence: Yaniv Dotan, 4116 Kilmer Avenue, Allentown, PA 18104, USA. E-mail: yaniv.dotandasluhn.org

\section{ABSTRACT}

Background: Idiopathic pulmonary fibrosis (IPF) is characterised by constant threat of acute exacerbation of IPF (AE-IPF). It would be significant to identify risk factors of AE-IPF. We sought to determine the prognostic value of lung transplantation candidacy testing for AE-IPF and describe explant pathology of recipients with and without AE-IPF before lung transplantation.

Methods: Retrospective cohort study of 89 IPF patients listed for lung transplantation. Data included pulmonary function testing, echocardiography, right heart catheterisation, imaging, oesophageal $\mathrm{pH} /$ manometry and blood tests. Explanted tissue was evaluated by pulmonary pathologists and correlated to computed tomography (CT) findings.

Results: Out of 89 patients with IPF, 52 were transplanted during stable IPF and 37 had AE-IPF before transplantation $(n=28)$ or death $(n=9)$. There were no substantial differences in candidacy testing with and without AE-IPF. AE-IPF had higher rate of decline of forced vital capacity (FVC) ( $21 \pm 22 \%$ versus $4.8 \pm 14 \%, \mathrm{p}=0.00019)$. FVC decline of $>15 \%$ had a hazard ratio of 7.2 for developing AE-IPF compared to FVC decline of $<5 \%$ ( $\mathrm{p}=0.004)$. AE-IPF had more secondary diverse histopathology ( $82 \%$ versus $29 \%$, $\mathrm{p}<0.0001)$ beyond diffuse alveolar damage. There was no correlation between ground-glass opacities (GGO) on chest CT at any point to development of AE-IPF ( $\mathrm{p}=0.077$ ), but GGO during AE-IPF predicted secondary pathological process beyond diffuse alveolar damage.

Conclusions: Lung transplantation candidacy testing including reflux studies did not predict AE-IPF besides FVC absolute decline. CT did not predict clinical or pathological AE-IPF. Secondary diverse lung pathology beyond diffuse alveolar damage was present in most AE-IPF, but not in stable IPF.

@ERSpublications

Transplant candidacy testing fails to predict acute exacerbation of IPF besides FVC absolute decline. Patients transplanted during acute exacerbation of IPF reveal multiple secondary lung histopathological processes beyond the expected DAD. https://bit.ly/3e1CPjO

Cite this article as: Dotan Y, Shapiro WB, Male E, et al. Clinical predictors and explant lung pathology of acute exacerbation of idiopathic pulmonary fibrosis. ERJ Open Res 2020; 6: 00261-2019 [https://doi.org/10.1183/23120541.00261-2019].

This article has supplementary material available from openres.ersjournals.com.

Received: 23 Sept 2019 | Accepted after revision: 10 June 2020

Copyright $\odot$ ERS 2020. This article is open access and distributed under the terms of the Creative Commons Attribution Non-Commercial Licence 4.0. 


\section{Introduction}

Idiopathic pulmonary fibrosis (IPF) is an interstitial lung disease (ILD) characterised by progressive parenchymal lung fibrosis, progressive dyspnoea and death with average survival of 3-5 years from the time of diagnosis [1, 2]. Many patients with IPF will experience a life-threatening acute exacerbation (AE-IPF). The annual risk of AE-IPF is $4-20 \%$, with an expected median survival of $<3$ months, $50 \%$ in-hospital mortality and up to $90 \%$ mortality for patients with AE-IPF and respiratory failure requiring mechanical ventilation [1, 3, 5-]. In 2007, the IPF Clinical Trials Network defined AE-IPF as an acute, clinically significant deterioration of unidentifiable cause in a patient with underlying IPF [6]. To fulfil the definition, patients should have previous diagnosis of IPF, unexplained worsening or development of dyspnoea within 30 days, high-resolution computed tomography (HRCT) with new bilateral ground-glass opacities (GGO) and/or consolidation superimposed on a background reticular or honeycomb pattern consistent with usual interstitial pneumonia (UIP) pattern, no evidence of pulmonary infection by endotracheal aspirate or bronchoalveolar lavage (BAL), and exclusion of alternative causes, including the following: congestive heart failure (CHF), pulmonary embolism and identifiable cause of acute lung injury [6]. In the Sildenafil Trial of Exercise Performance in IPD (STEP-IPF) trial, a post hoc analysis of respiratory events demonstrated that the incidence of AE-IPF was four per 100 patient-years when strict adherence to the 2007 criteria was utilised. The incidence increased to 20 per 100 patient-years when patients with "suspected" AE-IPF were added [7]. In 2016, an international group defined AE-IPF as an acute, clinically significant respiratory deterioration characterised by evidence of new widespread alveolar abnormality [1]. In the revised definition, exclusion of infection or other triggers were no longer required for the diagnosis of AE-IPF, and the only qualifier was that the radiologic findings were not fully explained by the presence of CHF or fluid overload. This broader definition resulted in criteria that were more inclusive and feasible for clinicians and clinical trials who have struggled with the previous requirement of exclusion of causes, in particular respiratory infections. In addition, this definition highlights that there is little clinical or biological support to distinguish idiopathic from nonidiopathic respiratory events in IPF [8]. Indeed, data suggest that clinical features and outcomes in "idiopathic" AE-IPF are similar to those encountered with respiratory worsening triggered by external events [7].

Given the high mortality rate of AE-IPF, it would be of significant benefit to predict which IPF patients are more likely to suffer an acute exacerbation and to understand the underlying pathological process of AE-IPF. Pathologic data of AE-IPF are scarce due to the high procedural risk to patients during acute exacerbations. Low forced vital capacity $(\mathrm{FVC})$ is the most consistent risk factor for AE-IPF, but other reported risk factors are low total lung capacity (TLC), diffusing capacity of the lung for carbon monoxide $\left(D_{\mathrm{LCO}}\right)$, 6-min walk distance $(6 \mathrm{MWD})$, resting oxygen saturation, cardiac disease, pulmonary hypertension and occult aspiration due to gastro-oesophageal reflux disease (GORD) $[1,3,6,7,9,14-]$.

In our study, we sought to determine the aetiology and risk factors for AE-IPF of a highly selected group of IPF patients based on lung transplantation candidacy testing and their explant lung pathology.

\section{Methods}

\section{Study population}

The study population included 89 patients with IPF listed for lung transplantation at Temple University Hospital (TUH; Philadelphia, PA, USA), from 2012 to 2016 (figure 1). Patients were diagnosed with IPF

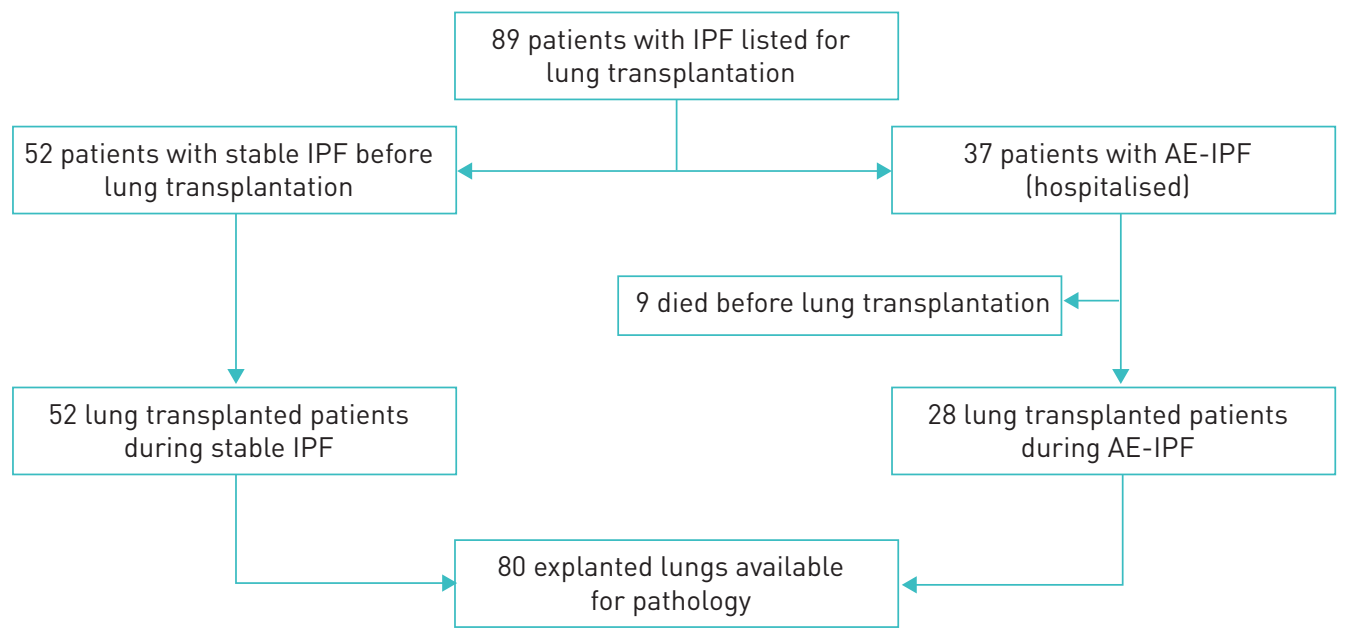

FIGURE 1 Study design. IPF: idiopathic pulmonary fibrosis; AE-IPF: acute exacerbation of IPF. 
by a multidisciplinary team, after exclusion of other known causes of ILD, blood testing and the presence of a UIP pattern on pre-lung transplantation HRCT [15]. AE-IPF was defined as an acute, clinically significant respiratory deterioration characterised by evidence of new widespread alveolar abnormality, not fully explained by the presence of CHF or fluid overload [1]. The patients' lung allocation score during listing and day of transplantation were recorded from the United Network of Organ Sharing.

\section{Pre-lung transplantation evaluation data}

All patients were evaluated for lung transplantation candidacy according to International Society for Heart and Lung Transplantation guidelines [16]. As a part of the evaluation for lung transplantation, patients undergo comprehensive blood tests, pulmonary function tests, 6-min walk test, echocardiography, right heart catheterisation (RHC), HRCT and clinical evaluation. As a routine part of our evaluation for lung transplantation of patients with ILD, all patients underwent 24-h oesophageal multichannel intraluminal impedance and $\mathrm{pH}$ monitoring for the detection of all types of reflux events within the oesophagus. This study distinguishes between acid reflux and non-acid reflux events, and detects correlations between reflux events and symptoms. Results were taken directly from the study report of each patient. 18 out of 37 patients with IPF hospitalised due to AE-IPF before completing full evaluation for lung transplantation did not complete selected tests (oesophageal $\mathrm{pH} /$ manometry) due to respiratory or haemodynamic instability and were excluded from the specific relevant analysis. Data were captured by chart review performed by YD, WBS, EM and ECD. Definitions for IPF, AE-IPF and pathologic diagnosis can be found in supplementary table S1. HRCT findings were captured by chart review and then reviewed by a thoracic radiologist $(\mathrm{CD})$ for this study. Time 0 was date of listing for lung transplantation. Other significant timings were date of AE-IPF, date of hospitalisation for AE-IPF, date of hospitalisation for lung transplantation, date of lung transplantation and date of death.

\section{Pathology}

All fixed and frozen tissues were obtained as part of the Lung Tissue Research Consortium (LTRC). The LTRC is sponsored by the American National Heart, Lung, and Blood Institute and TUH is one of the five clinical centres for LTRC in the USA. The LTRC protocol was utilised in all patients per the institutional review board protocol. Our clinical pathologist (AA) provided the reading. The tissue was prepared according to LTRC protocol and included only the lung explants. The tissues were preserved within $30 \mathrm{~min}$ of removal from the patients. Briefly, tissues were placed in liquid nitrogen, and, once completely frozen, transferred to a container filled with dry ice or a $-80^{\circ} \mathrm{C}$ freezer. Then it was covered with RNAlater (Ambion Inc., Austin, TX, USA) and totally immersed in fixative with $10 \%$ neutral buffered formalin. Slides were made with haematoxylin and eosin and pentachrome staining from the formalin-fixed specimens from each lobe. Sections were visually scored at $10 \times$ magnification by a certified pulmonary pathologist. Pathologic findings were captured by chart review.

\section{Statistical analysis}

Data are presented as mean $\pm \mathrm{SD}$ for continuous variables or percentages for categorical variables. Chi-squared was used for categorical data, and the paired or unpaired t-test was used for continuous data. Fisher's exact test was used for simple between-group comparisons. The Spearman rank-order correlation coefficient was used to measure the strength and direction of association between variables. A univariate Cox proportional hazards model was estimated to test the association between decline in FVC \% predicted from baseline and acute exacerbation. Missing data were considered missing at random and handled by omitting the cases with the missing data and analysing the remaining data for the specific analysis. p-values $<0.05$ were considered statistically significant. All data were analysed using SPSS (version 12.0; SPSS Inc., Chicago, IL, USA).

This study was conducted in accordance with the amended Declaration of Helsinki. The TUH review board approved the protocol, and written informed consent was obtained from all patients (protocol number 24473).

\section{Results}

\section{Study population}

89 patients with IPF were included in the analysis. Of the 89 patients, 52 had lung transplantation during stable disease and 37 patients had AE-IPF. Of the 37 patients with AE-IPF, 28 were hospitalised with AE-IPF before receiving lung transplantation and nine patients died during AE-IPF before receiving lung transplantation (figure 1). All 89 patients had pre-lung transplantation evaluation data available, and 80 patients had explant pathology. There were no statistically significant differences in baseline characteristics including age, sex, body mass index, number of comorbidities (including hypertension, diabetes mellitus, coronary artery disease and others) and smoking history between patients with and 
without AE-IPF (table 1). None of the patients were actively smoking or using nicotine for 6 months prior to transplantation candidacy evaluation or during active candidacy as confirmed by blood and urine nicotine and cotinine testing.

\section{Pre-lung transplantation evaluation data}

There were no statistically significant differences in baseline pulmonary function tests, 6MWD, oxygen saturation and GORD studies between patients with and without AE-IPF (table 1). Of note, the usage of anti-acid medications (proton pump inhibitors and/or histamine receptor blockers) was similar between the groups (AE-IPF group 70\%, no AE-IPF group 67\%). The AE-IPF group had lower serum albumin $\left(3 \pm 0.54\right.$ versus $\left.3.4 \pm 0.53 \mathrm{~g} \cdot \mathrm{dL}^{-1}, \mathrm{p}<0.001\right)$ and higher mean pulmonary artery pressure (mPAP; $27 \pm 11$

TABLE 1 Baseline characteristics and pre-evaluation data at time of lung transplantation

listing for patients without acute exacerbation of idiopathic pulmonary fibrosis (AE-IPF) before lung transplantation and patients with AE-IPF before lung transplantation

\begin{tabular}{|c|c|c|c|}
\hline & No AE-IPF group & AE-IPF group & p-value \\
\hline Subjects & 52 & 37 & \\
\hline \multicolumn{4}{|l|}{ Baseline characteristics } \\
\hline Age & $66 \pm 7$ & $67 \pm 5$ & 0.42 \\
\hline Male/female & $39 / 13$ & $22 / 15$ & 0.17 \\
\hline $\mathrm{BMI} \mathrm{kg} \cdot \mathrm{m}^{-2}$ & $28 \pm 4$ & $28 \pm 4$ & 0.28 \\
\hline \multicolumn{4}{|l|}{ Comorbidities and smoking history } \\
\hline Diabetes mellitus & $11(21)$ & 12 (32) & 0.33 \\
\hline Hypertension & $18(35)$ & $21(57)$ & 0.051 \\
\hline Coronary artery disease & $25(48)$ & $22(59)$ & 0.39 \\
\hline Congestive heart failure (systolic) & 0 & 1 (3) & 0.42 \\
\hline Smoking history & $38(73)$ & $25(68)$ & 0.65 \\
\hline Ever-smoker pack-years & $23 \pm 11$ & $16 \pm 29$ & 0.42 \\
\hline \multicolumn{4}{|l|}{ Pulmonary function tests } \\
\hline $\mathrm{FEV}_{1} / \mathrm{FVC}$ & $84 \pm 7$ & $86 \pm 8$ & 0.17 \\
\hline $\mathrm{FEV}_{1} \%$ pred & $52 \pm 15$ & $50 \pm 15$ & 0.42 \\
\hline FVC \% pred & $47 \pm 13$ & $43 \pm 12$ & 0.2 \\
\hline TLC \% pred & $48 \pm 9$ & $46 \pm 9$ & 0.41 \\
\hline RV \% pred & $44 \pm 15$ & $33 \pm 10$ & 0.004 \\
\hline$D_{\text {Lco }} \%$ pred & $28 \pm 11$ & $24 \pm 10$ & 0.17 \\
\hline Baseline oxygen saturation $\%$ & $90 \pm 6$ & $88 \pm 6$ & 0.09 \\
\hline 6-min walk distance $\mathrm{m}$ & $254 \pm 99$ & $218 \pm 74$ & 0.08 \\
\hline Lowest oxygen saturation \% & $83 \pm 13$ & $81 \pm 6$ & 0.61 \\
\hline \multicolumn{4}{|l|}{ Blood tests } \\
\hline Creatinine $\mathrm{mg} \cdot \mathrm{dL}^{-1}$ & $0.91 \pm 0.2$ & $0.82 \pm 0.3$ & 0.096 \\
\hline Albumin $\mathrm{g} \cdot \mathrm{dL}^{-1}$ & $3.4 \pm 0.53$ & $2.95 \pm 0.54$ & $0.0002^{9}$ \\
\hline WBC cells $\cdot \mathrm{mm}^{-3}$ & $12.8 \pm 6.5$ & $14 \pm 5$ & 0.23 \\
\hline Platelets cells $\cdot \mathrm{mm}^{-3}$ & $222 \pm 71$ & $212 \pm 113$ & 0.63 \\
\hline$P_{\mathrm{CO}_{2}} \mathrm{mmHg}$ & $43 \pm 9$ & $44 \pm 11$ & 0.54 \\
\hline \multicolumn{4}{|l|}{ Right heart catheterisation data } \\
\hline $\mathrm{mPAP} \mathrm{mmHg}$ & $23 \pm 8$ & $27 \pm 11$ & $0.028^{\Uparrow}$ \\
\hline PCWP mmHg & $8 \pm 4$ & $7.5 \pm 5$ & 0.46 \\
\hline Cardiac index $\mathrm{L} \cdot \mathrm{min}^{-1} \cdot \mathrm{m}^{-2}$ & $3 \pm 2.7$ & $2.7 \pm 0.7$ & 0.53 \\
\hline PVR WU & $3.4 \pm 2.6$ & $4.4 \pm 2.9$ & 0.11 \\
\hline \multicolumn{4}{|l|}{ Oesophageal $\mathrm{pH}$ and manometry data } \\
\hline Number of total reflux episodes $\#$ & $36 \pm 25$ & $30 \pm 26$ & 0.34 \\
\hline Time with $\mathrm{pH}<4^{\#} \%$ & $16 \pm 25$ & $17 \pm 24$ & 0.96 \\
\hline GORD score ${ }^{\#}$ & $10 \pm 15$ & $9 \pm 13$ & 0.76 \\
\hline \multicolumn{4}{|c|}{ 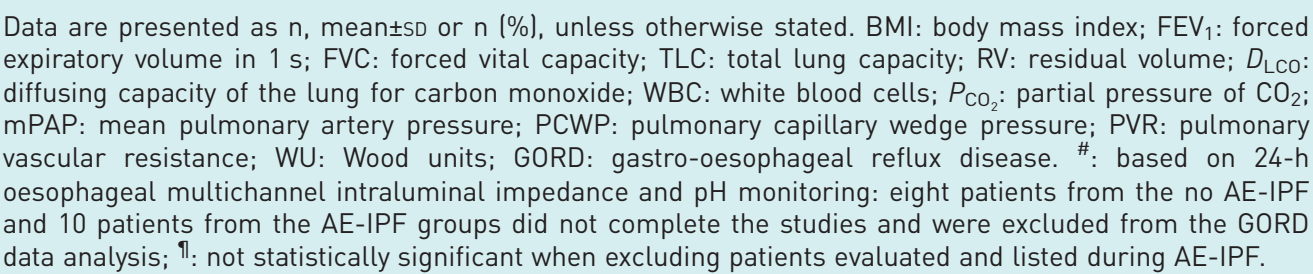 } \\
\hline
\end{tabular}




\begin{tabular}{|c|c|c|c|c|}
\hline & No AE-IPF & AE-IPF & AE-IPF HR $(95 \% \mathrm{CI})$ & $\mathrm{p}$-value \\
\hline $\begin{array}{l}\text { Subjects }^{\#} \\
\text { Absolute FVC de }\end{array}$ & 48 & 22 & & \\
\hline$<5 \%$ pred & $26(54)$ & $4(18)$ & Reference & \\
\hline $5-10 \%$ pred & $12(25)$ & 5 (23) & $3.2(0.77-13.5)$ & 0.11 \\
\hline $10-15 \%$ pred & $4(8)$ & $5(23)$ & $5.6(1.33-23.8)$ & 0.019 \\
\hline$>15 \%$ pred & 6 (13) & $8(34)$ & $7.3(1.91-27.6)$ & 0.004 \\
\hline
\end{tabular}

versus $23 \pm 8 \mathrm{mmHg}, \mathrm{p}=0.028)$ measured by $\mathrm{RHC}$. However, when excluding patients who were urgently evaluated during AE-IPF, there were no statistically significant differences in albumin blood level or mPAP (table 1).

\section{Lung function decline}

Patients with AE-IPF had higher absolute decline of FVC compared to patients without AE-IPF before lung transplantation $(21 \pm 22 \%$ versus $4.8 \pm 14 \%, \mathrm{p}=0.00019)$. The hazard ratio for developing AE-IPF was higher as FVC decline increased: for patients with absolute decline of 5-10\%, hazard ratio was 3.2 (nonsignificant) to develop AE-IPF when compared to patients who had $<5 \%$ absolute decline. Similarly, patients with absolute decline of $10-15 \%$ and $>15 \%$ had hazard ratios $5.6(p=0.019)$ and $7.3(p=0.004)$, respectively, to develop AE-IPF when compared to patients who had $<5 \%$ absolute decline (table 2 ).

\section{Pathology data}

Explanted lungs for pathology studies were available for all 52 patients who were transplanted during stable IPF and 28 patients who were transplanted during AE-IPF. Nine patients died during AE-IPF after listing and before receiving lung transplantation. The pathology from 37 (71\%) out of 52 patients without AE-IPF showed UIP alone. The pathology from the other 15 (29\%) patients had at least one secondary pattern including cryptogenic organising pneumonia $(C O P, n=5)$, desquamative interstitial pneumonia (DIP, $n=4$ ), combined patterns (i.e. COP and DIP, $n=3$ ), acute nonspecific inflammation $(n=2)$ and diffuse alveolar damage (DAD, $n=1)$. Of the 28 patients with AE-IPF, five (18\%) had only UIP on pathology and $23(82 \%)$ had at least one secondary pattern including acute nonspecific inflammation $(n=7)$, DIP $(n=6)$, $\operatorname{DAD}(n=4)$, combined patterns (i.e. COP and DIP, $n=4)$ and only COP $(n=2)$ (figure 2 ).

a)

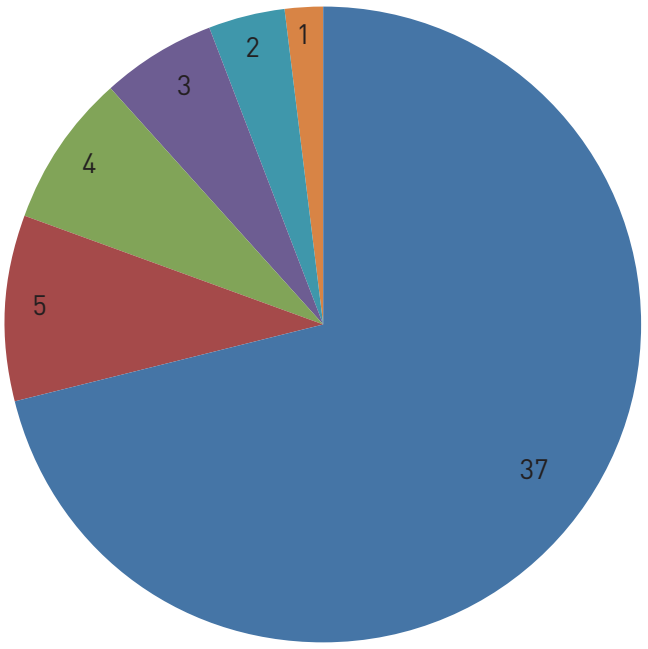

b)

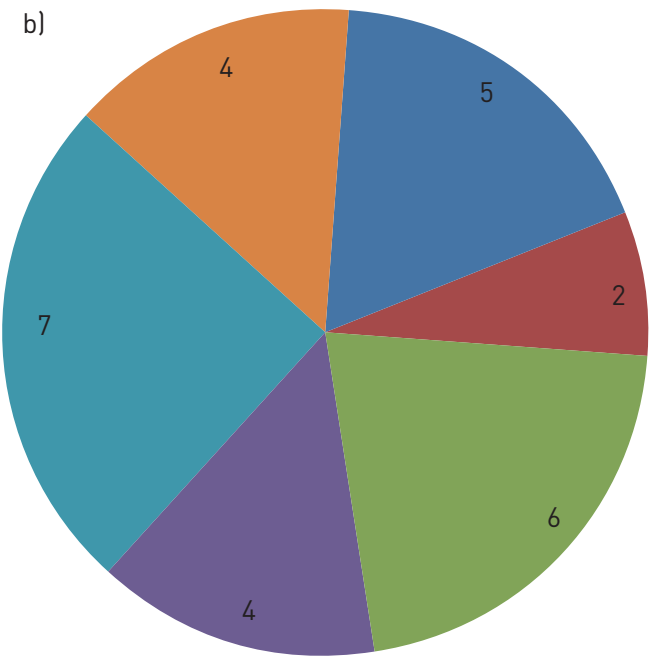

UIP only

$\mathrm{UIP}+\mathrm{COP}$

UIP+DIP

UIP $+>1$ pattern

UIP+acute nonspecific inflammation

UIP+DAD

FIGURE 2 Prevalence of explanted lung pathology patterns in patients a) without ( $n=52$ ) and b) with ( $n=28$ ) acute exacerbation of idiopathic pulmonary fibrosis (AE-IPF). UIP: usual interstitial pneumonia; COP: cryptogenic organising pneumonia; DIP: desquamative interstitial pneumonia; DAD: diffuse alveolar damage. 
TABLE 3 High-resolution computed tomography (HRCT) findings, timing, ground-glass opacities (GGO) and correlation to pathologic findings in both groups

\begin{tabular}{|c|c|c|}
\hline & No AE-IPF & AE-IPF \\
\hline Subjects & 52 & 37 \\
\hline Baseline HRCT UIP pattern (typical/probable/indeterminate) & $33 / 14 / 5$ & $25 / 9 / 3$ \\
\hline GGO severity in chest HRCT at any time scored by thoracic radiologist (mild/moderate/severe ${ }^{\#}$ ) & $5 / 2 / 10$ & $3 / 0 / 16$ \\
\hline Had chest HRCT 30 days prior to lung transplantation & $2 / 52(4)$ & $13 / 28^{\text {Tी }}(46)$ \\
\hline Had GGO in chest HRCT 30 days prior to lung transplantation & $1 / 2(50)$ & $9 / 13(69)$ \\
\hline
\end{tabular}

HRCT and explant pathology correlation

All patients had at least one pre-lung transplantation HRCT scan during the evaluation process and/or waiting period. UIP pattern was defined as typical, probable or indeterminate for UIP [17]. Of the 89 patients, 58 had typical, 23 had probable and eight had indeterminate UIP pattern. Out of 52 patients without AE-IPF, 17 (33\%) had GGO at some point prior to transplantation, and $35(67 \%)$ did not have any GGO. Of the 37 patients with AE-IPF, 19 (51\%) had GGO at some point, and 18 (49\%) did not have any GGO ( $\mathrm{p}=0.077)$. All patients without AE-IPF $(\mathrm{n}=52)$ had both pre-lung transplantation HRCT and explant lung pathology. 28 out of 37 patients with AE-IPF had both pre-lung transplantation chest HRCT and explant lung pathology. In order to correlate between the explant histopathology and concurrent chest HRCT we compared the available computed tomography images and histopathology for all patients in whom the HRCT was performed within 30 days prior to lung transplantation. 15 patients from both groups underwent chest HRCT within 30 days before lung transplantation: two patients from the no AE-IPF group, and 13 patients from the AE-IPF group. One of the two patients from the no AE-IPF had GGO in HRCT and no secondary pathological process. Nine of the 13 patients of the AE-IPF had GGO in HRCT and all had secondary pathological process. All data are summarised in table 3.

\section{Timing of AE-IPF, listing and lung transplantation or death}

Patients without AE-IPF $(n=52)$ were hospitalised once a donation was available. Their hospitalisation was brief and in most cases they received lung transplantation within a day. Patients with AE-IPF ( $\mathrm{n}=37)$ were hospitalised for acute or chronic respiratory failure. Some of them $(n=18)$ were listed previously and others $(n=19)$ were urgently listed during that hospitalisation. Nine of the 37 patients with AE-IPF died before receiving lung transplantation. Timing of these events are summarised in table 4.

\section{AE-IPF microbiological data}

Of the 28 patients who had AE-IPF before transplantation, 12 had sputum bacterial culture before mechanical ventilation (all negative cultures); 11 patients had viral cultures before mechanical ventilation

TABLE 4 Timing of listing, hospitalisation and lung transplantation or death for both groups

\begin{tabular}{|c|c|c|}
\hline & No AE-IPF & AE-IPF \\
\hline Subjects $n$ & 52 & 37 \\
\hline Time from hospitalisation to lung transplantation" days & $0.4 \pm 0.5$ & $21.6 \pm 24$ \\
\hline Time from listing to transplantation days & $164+175$ & $\begin{array}{l}\text { Listed during AE-IPFף } \\
20 \pm 30.8 \\
\text { Listed before AE-IPFף } \\
167 \pm 146\end{array}$ \\
\hline $\begin{array}{l}\text { Time from AE-IPF to death before getting lung } \\
\text { transplantation }(n=9) \text { days }\end{array}$ & NA & $37.8 \pm 32.7$ \\
\hline Time from lung transplantation to discharge days & $15.9+14.7$ & $31.9 \pm 28.6$ \\
\hline
\end{tabular}




\begin{tabular}{|c|c|c|}
\hline & Patients examined & Positive results \\
\hline Sputum bacterial culture & 18 & $4^{\#}(22)$ \\
\hline Sputum viral culture or PCR & 17 & $1(6)$ \\
\hline
\end{tabular}

(all negative); and three patients had viral respiratory PCR sample before mechanical ventilation (one positive for rhinovirus). Of the nine patients with AE-IPF who died before transplantation, seven patients had sputum bacterial culture before mechanical ventilation, with four positive cultures (three bacterial and one fungal), and none had viral culture or PCR data (table 5).

\section{Discussion}

The main findings of our study are as follows. 1) Transplantation candidate testing including GORD studies were not associated with AE-IPF; 2) patients with higher absolute FVC decline had increased risk of developing AE-IPF; 3) AE-IPF was characterised by histopathological UIP and a diverse secondary pathologic diagnosis identified in explanted lung tissue; 4) HRCT UIP patterns and presence or absence of GGO were not associated with clinical AE-IPF, but GGO in HRCT during AE-IPF correlated with secondary pathologic diagnosis beyond UIP; and 5) patients with IPF without AE who had UIP and a secondary pathologic diagnosis could not be distinguished clinically prior to transplantation.

Given the high rate of mortality of AE-IPF, it would be of significant benefit to predict which IPF patients are more likely to suffer an acute exacerbation. Previously reported risk factors for AE-IPF included low FVC, TLC, $D_{\mathrm{LCO}}, 6 \mathrm{MWD}$, resting oxygen saturation, cardiac disease and pulmonary hypertension. Of these, FVC is the most consistent parameter $[1,6,9,10,18]$. In our study, we found that patients with AE-IPF had increased absolute decline of FVC. We found that higher FVC \% predicted absolute decline had increased hazard ratio for developing AE-IPF: the risk for an AE was 7.3-fold higher in patients with $>15 \%$ absolute FVC decline compared to patients with $<5 \%$ absolute FVC decline. This increased absolute decline can potentially be a predictor of rapid subclinical deterioration leading to clinical respiratory failure and hospitalisation for AE-IPF. Since the mortality of AE-IPF with respiratory failure is up to $90 \%$ for patients with $\mathrm{AE}-\mathrm{IPF}$ and respiratory failure requiring mechanical ventilation $[1,3,5-]$, fastening the process of evaluation and early listing for patients with rapid decline of FVC might prevent lung transplantation during AE-IPF, which has been shown by our group to have worse short- and long-term outcomes [19].

In our study, none of the other parameters checked were statistically significant in predicting AE-IPF. We did find that residual lung volume was lower at baseline in those who had AE-IPF, which has not been reported previously in the literature ( $44 \pm 15 \%$ pred versus $33 \pm 10 \%$ pred). However, the significance of this finding is unknown. The other two parameters that were statistically different between the groups were mPAP and albumin. However, these parameters lost statistical significance when AE-IPF patients who were urgently evaluated during the hospitalisation were excluded.

The cause of AE-IPF remains elusive. Proposed aetiological factors include intrinsic acceleration of the fibrotic disease or acute injury in response to external stimuli (e.g. infection, micro-aspiration, trauma, etc.) [1]. However, existing studies examining clinical, molecular, genetic, post-mortem, BAL and environmental exposure have failed to find a consistent or common aetiology of AE-IPF [12, 20-23-]. We examined most components of pre-transplantation patient characterisation and failed to identify a risk factor for AE-IPF. The use of anti-acid therapy has been shown to be associated with fewer AE-IPF, suggesting that GORD contributes to disease progression [24]. We examined the use of anti-acid therapy and GORD severity scores in those patients with and without AE-IPF. The use of anti-acid therapy was similar in both groups. The GORD severity as defined by pre-transplantation oesophageal studies was similar between the two groups.

Reported surgical lung biopsies of hospitalised AE-IPF are limited. PARAMBIL et al. [25] described seven patients hospitalised for AE-IPF who underwent surgical lung biopsy after a median interval of 10 days following hospitalisation. All patients were receiving mechanical ventilation at the time of biopsy for a median of 8 days. They described a combination of UIP and DAD in five out of seven patients. One had DAD without UIP, although UIP and DAD were confirmed at autopsy. The other had biopsy histology of 
UIP and patchy zones of associated COP without features of DAD. Additional features of acute lung injury in the six patients with DAD included hyaline membranes $(n=5)$, squamous metaplasia of bronchiolar epithelium $(n=4)$ and fibrin thrombi $(n=1)$. None demonstrated nonspecific interstitial pneumonia (NSIP) or DIP.

Description of explant histopathology in IPF transplantation recipients is limited to two studies and a total of 42 lungs. Of these 42 patients, AE-IPF explant pathology is limited to one clinically diagnosed AE-IPF and seven with HRCTs read as possible AE-IPF. None were hospitalised prior to transplantation. KATZENSTEIN et al. [26] examined the histology of surgical lung biopsy and explanted lungs of 20 lung transplantation recipients at a single centre. None were reported to have been hospitalised prior to transplantation. Explant pathology showed UIP diagnosis in 19 and NSIP in one patient. 18 had UIP and focal or prominent "DIP-like reaction" and 16 had "NSIP-like areas". The cohort included several patients with connective tissue disease-related ILD and the entire cohort was quite young, perhaps younger than a modern IPF/transplantation cohort. RABEYRIN et al. [27] examined 22 explanted lungs from patients transplanted for IPF. All explants showed UIP, 16 showed chronic inflammation, 13 showed UIP with NSIP-like areas, four showed DIP-like areas and one showed DAD. Of note, DAD and COP were rare in both studies, occurring in only two and one out of 42 explants, respectively. If these patients had been listed for transplantation in the lung allocation score era the timing of the candidate listing and transplantation would probably have been very different. Modern lung transplantation programmatic attitudes and life-support technology allow for the survival and transplantation of much sicker patients who previously did not survive hospitalisation for AE-IPF. Most of the AE-IPF patients described in our article would not have received transplantation and explant histopathology, which might affect the results showing more advanced and severe pathologic findings.

The relative paucity of DAD in our AE-IPF cohort is contrary to the widely held assumption that AE-IPF common histopathology is DAD superimposed upon UIP. We speculate that AE-IPF has several endotypes including a more protracted subacute worsening of pre-existing severe disease leading to severe hypoxaemia that cannot be supported at home. This subacute exacerbation endotype may lack a discrete acute lung injury and therefore not have DAD histology. The striking increased proportion of associated secondary histopathology including DIP and COP in our AE-IPF versus stable IPF may differentiate progressive IPF headed for hospitalisation and advanced support from a more stable IPF. DIP is known to be caused by cigarette smoke exposure and typically manifests as acute or subacute respiratory failure. However, none of the patients were actively smoking during 6 months prior to candidate evaluation or after active listing for transplantation as confirmed by serum and urine nicotine and cotinine testing. There was no correlation of smoking burden (pack-years) with DIP pattern on lung pathology. Since air pollution including passive smoke exposure is known to exacerbate chronic obstructive lung disease and asthma, and given our findings, we can only speculate that poor air quality might potentially increase AE-IPF risk $[28,29]$.

Our study has several limitations. First, given that this is a single-centre study, our medical management, level of experience and surgical techniques cannot necessarily be generalised. Second, despite the sample size being larger than those of previous studies, the size is still small with inherently reduced statistical power. Third, pathologic sampling error is possible and may underestimate the degree of significant pathological changes, especially with disease that is patchy in nature. Fourth, since all patients who had AE-IPF before lung transplantation were treated with supportive measures including systemic steroids, and the time gap between AE-IPF to transplantation was $22 \pm 24$ days, pathologic conclusions should be cautious since systemic steroids have the potential to affect the findings, specifically the inflammatory response rather than fibrotic changes. Fifth, chest HRCT findings such as GGO are not specific to AE-IPF. GGO might represent other processes such as volume overload, infection, diffuse alveolar haemorrhage and more. Furthermore, the number of patients who had both HRCT in the 30 days before lung transplantation and explant lung pathology is small $(n=9)$ and conclusions regarding that are of limited value. Finally, we did not characterise the baseline and change in fibrotic HRCT extent, which are two important variables potentially influencing AE-IPF.

In summary, we found that among comprehensive transplantation candidacy testing, only absolute decline of FVC was a predictor of AE-IPF. We found that patients with AE-IPF had high frequency of secondary acute pathological process beyond UIP and DAD. These processes were diverse and seen also in patients with clinical stable disease. HRCT UIP patterns and presence or absence of GGO did not predict clinical or pathological AE-IPF, but GGO during AE-IPF did predict secondary pathological process beyond UIP. We conclude that AE-IPF might be a multifactorial process with similar clinical presentation. Emerging risk factors from our study might be exposure to cigarette smoking and/or air pollution, as reflected by the high frequency of DIP in explanted lungs of patients with AE-IPF. Further clinical-pathologic studies are needed to confirm our findings. 
Author contributions: Y. Dotan collected the data and is the guarantor of the article, taking responsibility for the integrity of the work as a whole from inception to published article. E. Male and E.C. Dominguez collected data. W.B. Shapiro helped write the manuscript. A. Aneja reviewed the pathology studies. Z. Huaqing conducted the statistical analysis. C. Dass reviewed the imaging studies. K. Shenoy, N. Marchetti, F.C. Cordova, G.J. Criner and A.J. Mamary were the transplantation pulmonologists for the patients included in the study, conducted the pre-transplantation evaluation, and treated the patients before and after lung transplantation. A.J. Mamary was a primary co-author, responsible for the study concept and helped write the manuscript.

Conflict of interest: None declared.

\section{References}

1 Collard HR, Ryerson CJ, Corte TJ, et al. Acute exacerbation of idiopathic pulmonary fibrosis. an international working group report. Am J Respir Crit Care Med 2016; 194: 265-275.

2 Natsuizaka M, Chiba H, Kuronuma K, et al. Epidemiologic survey of Japanese patients with idiopathic pulmonary fibrosis and investigation of ethnic differences. Am J Respir Crit Care Med 2014; 190: 773-779.

3 Song JW, Hong SB, Lim CM, et al. Acute exacerbation of idiopathic pulmonary fibrosis: incidence, risk factors and outcome. Eur Respir J 2011; 37: 356-363.

4 Al-Hameed FM, Sharma S. Outcome of patients admitted to the intensive care unit for acute exacerbation of idiopathic pulmonary fibrosis. Can Respir J 2004; 11: 117-122.

5 Ryerson CJ, Cottin V, Brown KK, et al. Acute exacerbation of idiopathic pulmonary fibrosis: shifting the paradigm. Eur Respir J 2015; 46: 512-520.

6 Collard HR, Moore BB, Flaherty KR, et al. Acute exacerbations of idiopathic pulmonary fibrosis. Am J Respir Crit Care Med 2007; 176: 636-643.

7 Collard HR, Yow E, Richeldi L, et al. Suspected acute exacerbation of idiopathic pulmonary fibrosis as an outcome measure in clinical trials. Respir Res 2013; 14: 73.

8 Hambly N, Cox G, Kolb M. Acute exacerbations of idiopathic pulmonary fibrosis: tough to define; tougher to manage. Eur Respir J 2017; 49: 1700811.

9 Kondoh Y, Taniguchi H, Katsuta T, et al. Risk factors of acute exacerbation of idiopathic pulmonary fibrosis. Sarcoidosis Vasc Diffuse Lung Dis 2010; 27: 103-110.

10 Judge EP, Fabre A, Adamali HI, et al. Acute exacerbations and pulmonary hypertension in advanced idiopathic pulmonary fibrosis. Eur Respir J 2012; 40: 93-100.

11 Reichmann WM, Yu YF, Macaulay D, et al. Change in forced vital capacity and associated subsequent outcomes in patients with newly diagnosed idiopathic pulmonary fibrosis. BMC Pulm Med 2015; 15: 167.

12 Lee JS, Song JW, Wolters PJ, et al. Bronchoalveolar lavage pepsin in acute exacerbation of idiopathic pulmonary fibrosis. Eur Respir J 2012; 39: 352-358.

13 Lee JS, Collard HR, Raghu G, et al. Does chronic microaspiration cause idiopathic pulmonary fibrosis? Am J Med 2010; 123: 304-311.

14 Raghu G, Freudenberger TD, Yang S, et al. High prevalence of abnormal acid gastro-oesophageal reflux in idiopathic pulmonary fibrosis. Eur Respir J 2006; 27: 136-142.

15 Raghu G, Collard HR, Egan JJ, et al. An official ATS/ERS/JRS/ALAT statement: idiopathic pulmonary fibrosis: evidence-based guidelines for diagnosis and management. Am J Respir Crit Care Med 2011; 183: 788-824.

16 Weill D, Benden C, Corris PA, et al. A consensus document for the selection of lung transplant candidates: 2014 - an update from the Pulmonary Transplantation Council of the International Society for Heart and Lung Transplantation. J Heart Lung Transplant 2015; 34: 1-15.

17 Raghu G, Remy-Jardin M, Myers JL, et al. Diagnosis of idiopathic pulmonary fibrosis. An official ATS/ERS/JRS/ ALAT clinical practice guideline. Am J Respir Crit Care Med 2018; 198: e44-e68.

18 Kondoh Y, Taniguchi H, Ebina M, et al. Risk factors for acute exacerbation of idiopathic pulmonary fibrosis extended analysis of pirfenidone trial in Japan. Respir Investig 2015; 53: 271-278.

19 Dotan Y, Vaidy A, Shapiro WB, et al. Effect of acute exacerbation of idiopathic pulmonary fibrosis on lung transplantation outcome. Chest 2018; 154: 818-826.

20 Kakugawa T, Sakamoto N, Sato S, et al. Risk factors for an acute exacerbation of idiopathic pulmonary fibrosis. Respir Res 2016; 17: 79.

21 Oda K, Ishimoto H, Yamada S, et al. Autopsy analyses in acute exacerbation of idiopathic pulmonary fibrosis. Respir Res 2014; 15: 109.

22 Johannson KA, Vittinghoff E, Lee K, et al. Acute exacerbation of idiopathic pulmonary fibrosis associated with air pollution exposure. Eur Respir J 2014; 43: 1124-1131.

23 Schupp JC, Binder H, Jäger B, et al. Macrophage activation in acute exacerbation of idiopathic pulmonary fibrosis PLoS One 2015; 10: e0116775.

24 Konishi K, Gibson KF, Lindell KO, et al. Gene expression profiles of acute exacerbations of idiopathic pulmonary fibrosis. Am J Respir Crit Care Med 2009; 180: 167-175.

25 Parambil JG, Myers JL, Ryu JH. Histopathologic features and outcome of patients with acute exacerbation of idiopathic pulmonary fibrosis undergoing surgical lung biopsy. Chest 2005; 128: 3310-3315.

26 Katzenstein AL, Zisman DA, Litzky LA, et al. Usual interstitial pneumonia: histologic study of biopsy and explant specimens. Am J Surg Pathol 2002; 26: 1567-1577.

27 Rabeyrin M, Thivolet F, Ferretti GR, et al. Usual interstitial pneumonia end-stage features from explants with radiologic and pathological correlations. Ann Diagn Pathol 2015; 19: 269-276.

28 Papiris SA, Kagouridis K, Kolilekas L, et al. The new idiopathic pulmonary fibrosis acute exacerbations document: one step ahead but still suspended in the air. Am J Respir Crit Care Med 2017; 195: 267-269.

29 Foreman MG, DeMeo DL, Hersh CP, et al. Clinical determinants of exacerbations in severe, early-onset COPD. Eur Respir J 2007; 30: 1124-1130. 